\title{
Activation of $\beta$-Catenin Signalling Enhances the Osteogenic Gene Response to Mechanical Loading in Mesenchymal Stem Cells
}

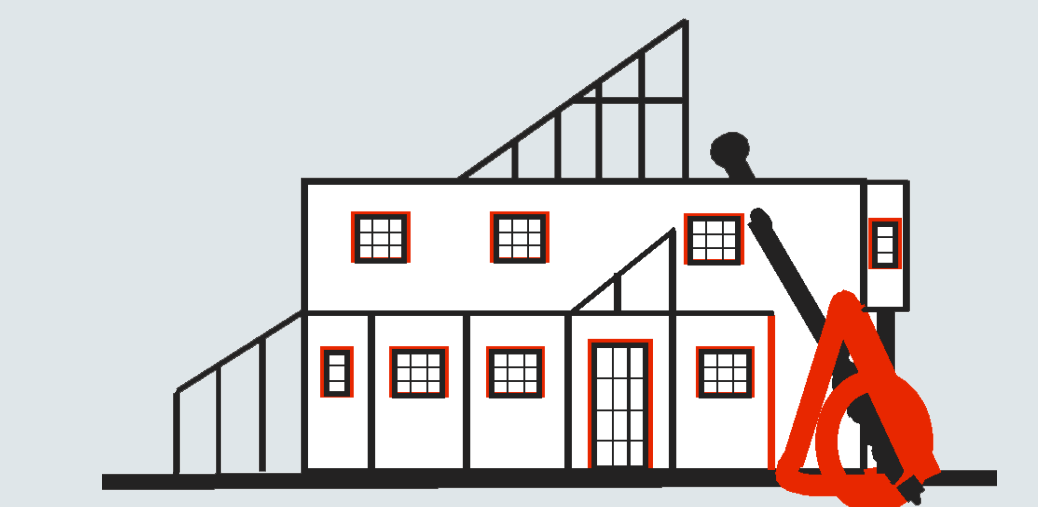

www.biomechanics.d

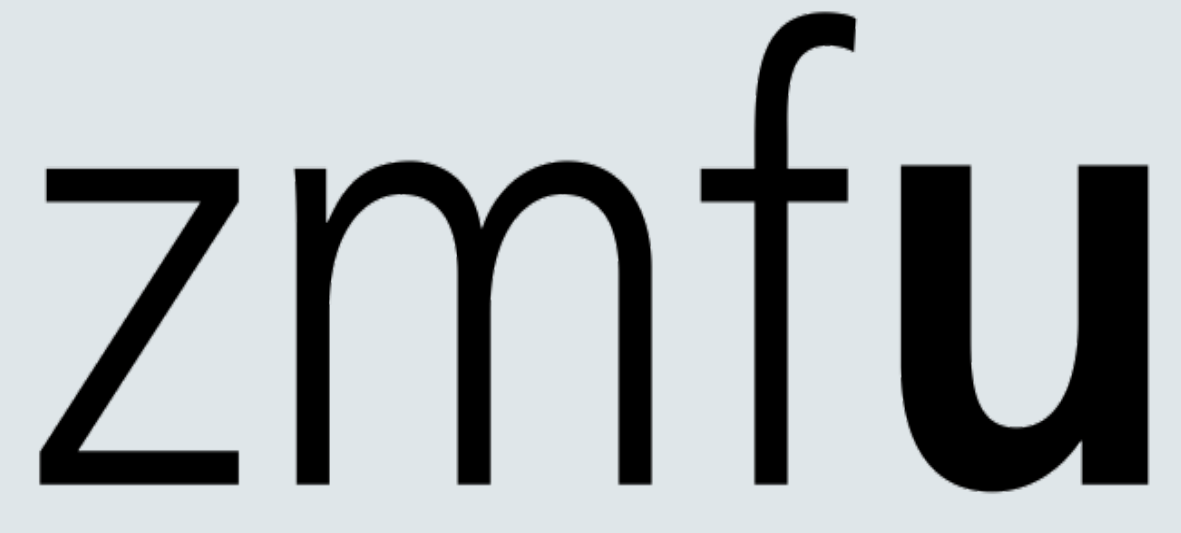

\section{Nemitz ${ }^{1}$, F. Jakob², A. Ignatius ${ }^{1}$, A. Liedert ${ }^{1}$}

${ }^{1}$ Institute of Orthopaedic Research and Biomechanics, Center of Musculoskeletal Research, University of Ulm, Germany

${ }^{2}$ Orthopedic Center for Musculoskeletal Research, University of Würzburg, Germany

\section{Introduction}

Clinical studies have demonstrated that aging and osteoporosis are associated with an increase of adipose tissue in the bone marrow [1]. Wnt/ $\beta$-catenin signalling and mechanical loading are able to inhibit adipogensis and to stimulate osteoblastogenesis of mesenchymal stem cells [2,3] The involvement of $\beta$-catenin signalling in mechanically induced bone formation has already been shown in vivo using a tibia loading model [4].

The aim of the present study was to investigate the influence of the activation of $\beta$ catenin and mechanical signalling on the osteogenic and adipogenic response of mesenchymal stem cells in vitro.

\section{Methods}

\section{Cell culture}

- Cells of the mesenchymal cell line C3H10T1/2 were seeded in collagene type I precoated silicone dishes and cultivated in adipogenic medium for 5 days, after precultivation in standard medium for 2 days.

Oil Red O staining

- Oil Red O staining was performed for detection of cytoplasmic lipid vacuoles.

Chemical and mechanical stimulation

$10 \mathrm{mM} \mathrm{LiCl}$ or $20 \mu \mathrm{M}$ SB415286 was added for activating $\beta$-catenin signalling. Control groups received the respective vehicle. Cells were loaded by daily homogenous cyclic stretching using sinusoidal strain at $2 \%$ and a frequency of $1 \mathrm{~Hz}$ for $0.5 \mathrm{~h}$ for 5 days. Control dishes were treated in parallel without mechanical stretching

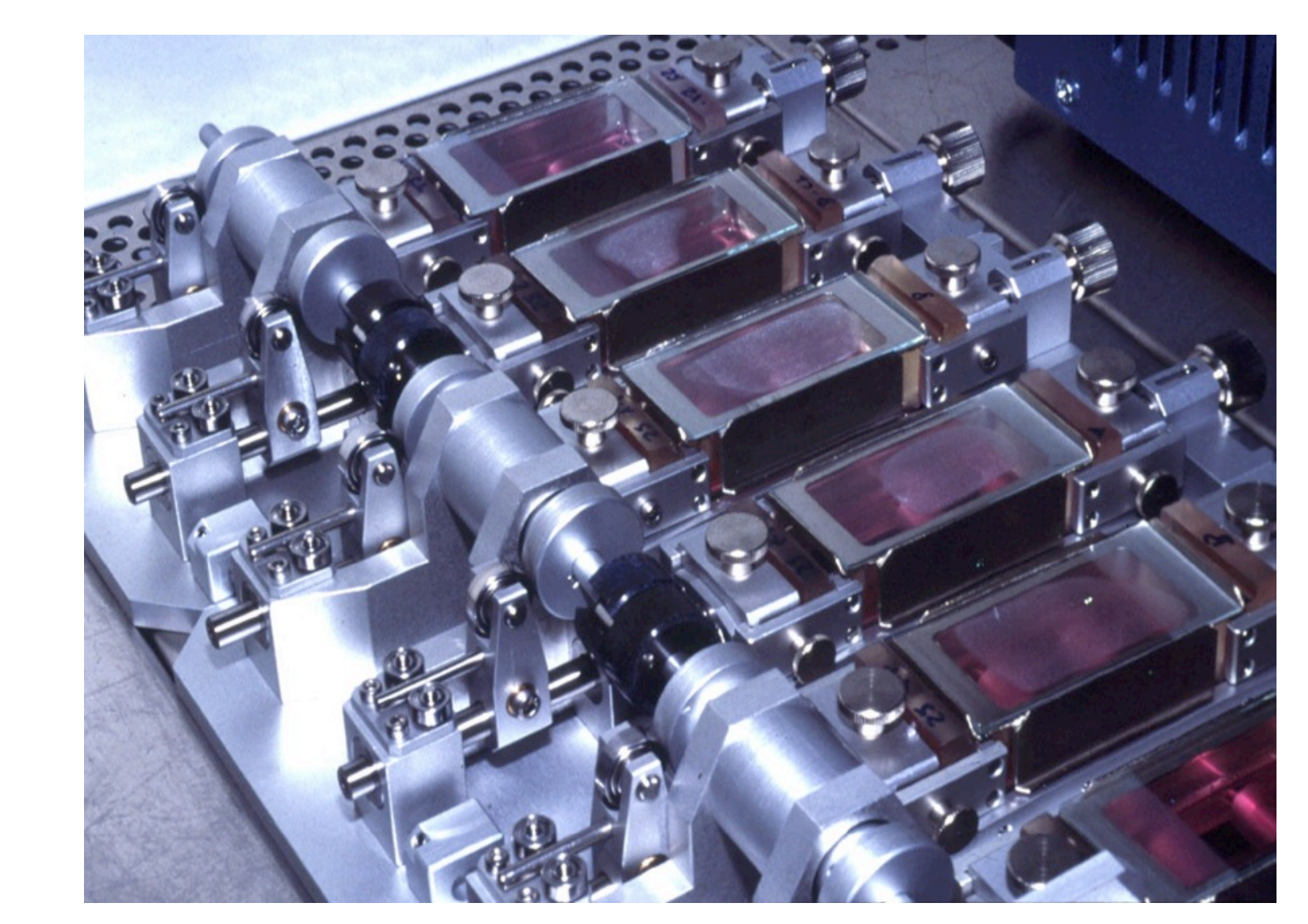

Real-time RT-PCR and Western blotting

- Cells were lysed immediately after the last stretching for total RNA isolation and real-time RT-PCR or for protein preparation and Western blotting.

Statistics

Three independent experiments in duplicate $(n=6)$ were performed. Data were analyzed for significance (value $p \leq 0.05$ ) using Student's $t$-test. The results were presented as mean value \pm standard deviation (SD).

\section{Results}

- Lipid vacuoles were remarkably reduced by $\mathrm{LiCl}$ or SB415286 as well as by mechanical loading. SB415286 treatment in addition to mechanical loading completely abolished lipid vacuole formation.
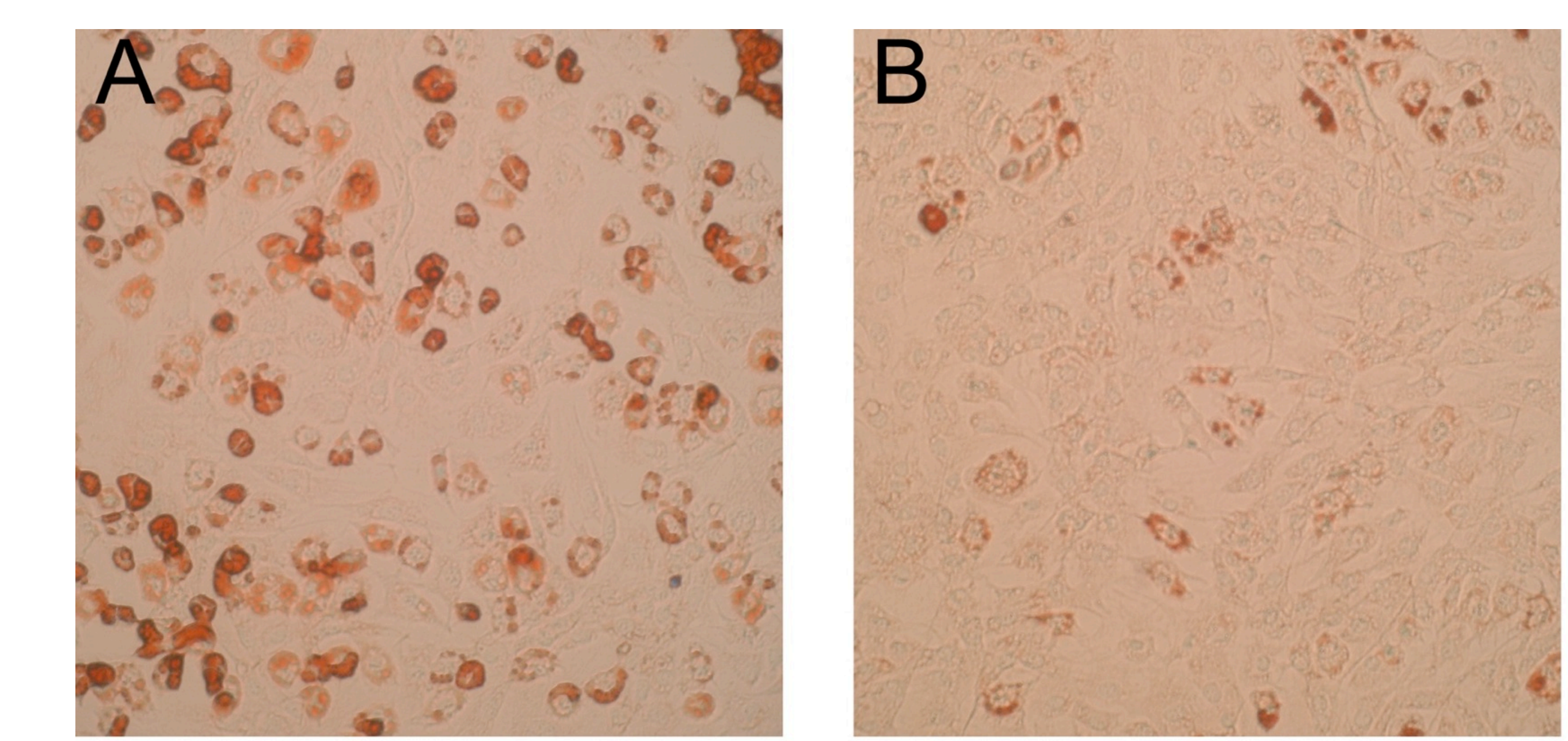

D
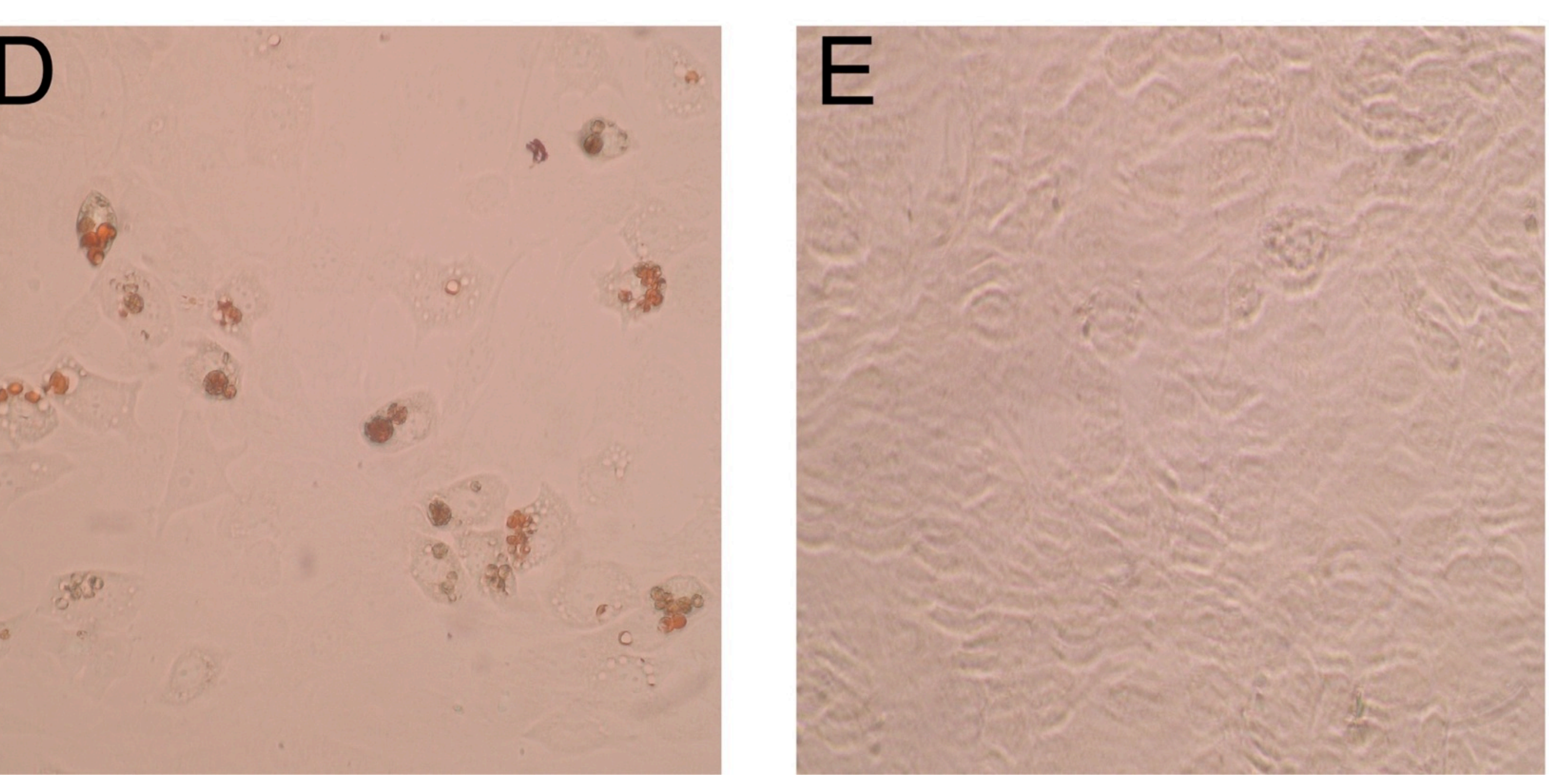

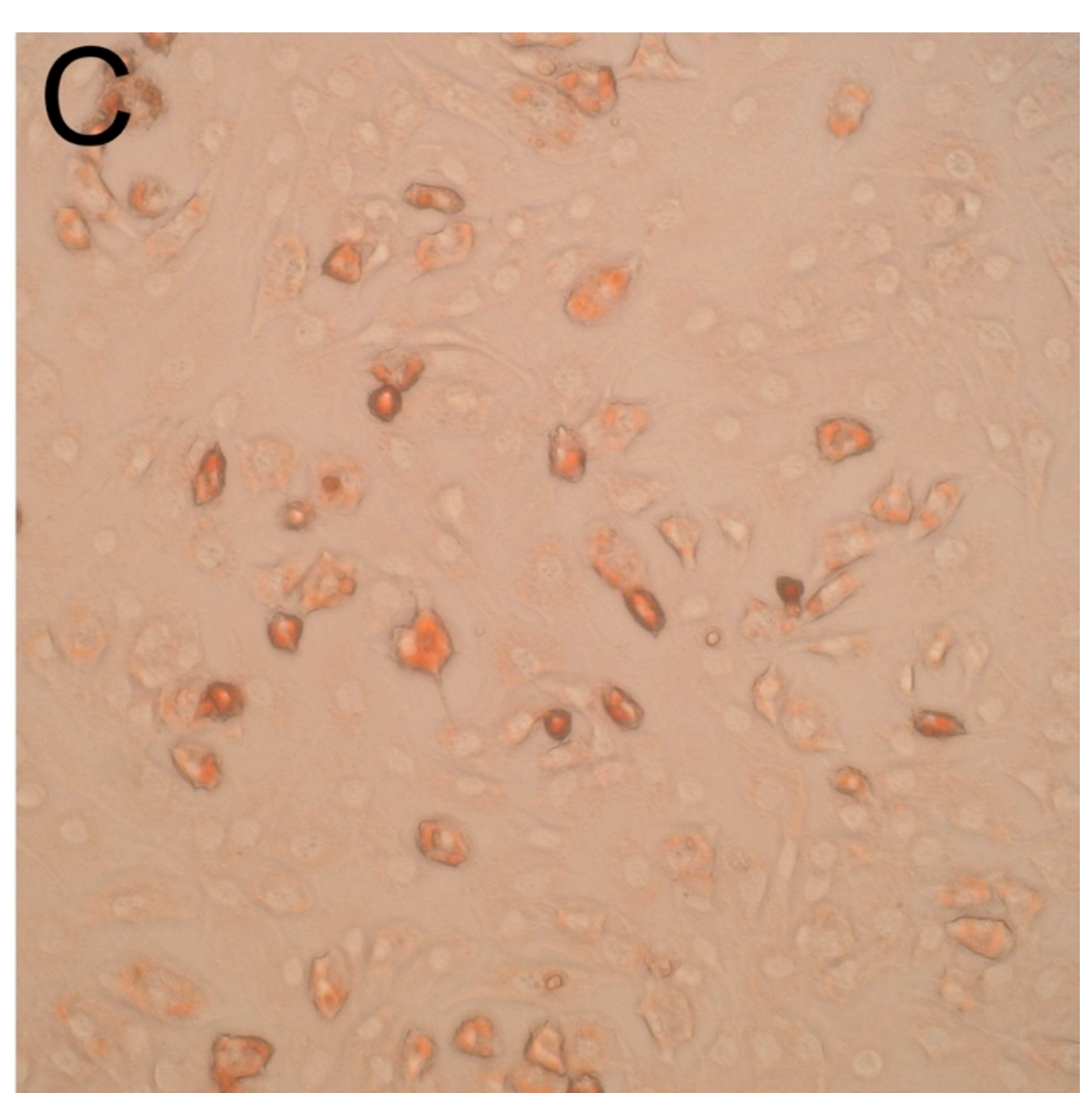

A: Control

B: $\mathrm{LiCl}$

C: SB415286

D: Mechanical loading

E: Mechanical loading + SB415286
- Mechanical loading and SB415286 upregulated the relative gene expression of the osteogenic markers Runx-2, Cox-2 and Cyr-61. SB415286 treatment in addition to mechanical loading enhanced the mechanically induced expression of Runx-2, Cox-2 and Cyr-61.
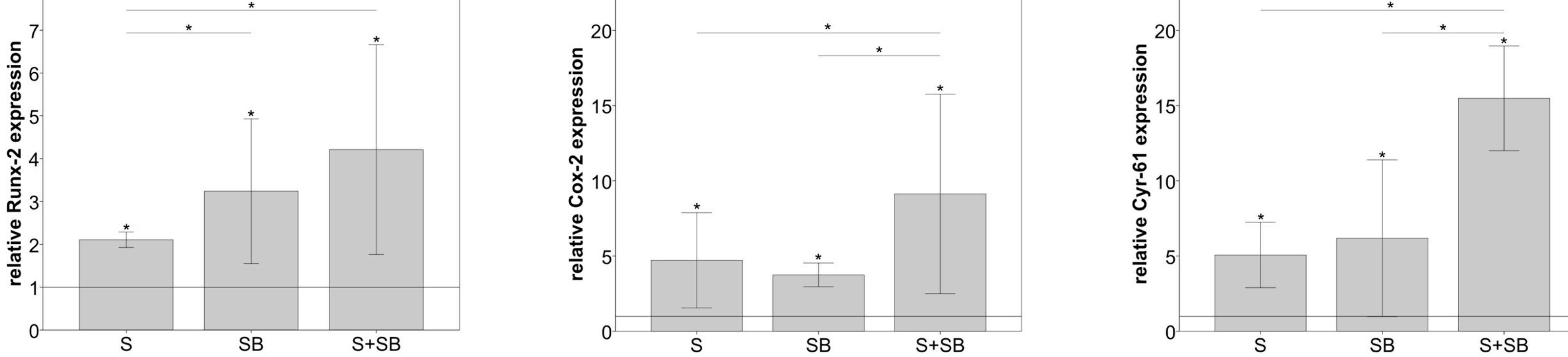

- The gene expression of the adipogenic markers Ppary and Cebpa was downregulated by mechanical loading and SB415286. SB415286 treatment had a sensitizing effect on mechanically reduced expression of Ppary and Cebpa.
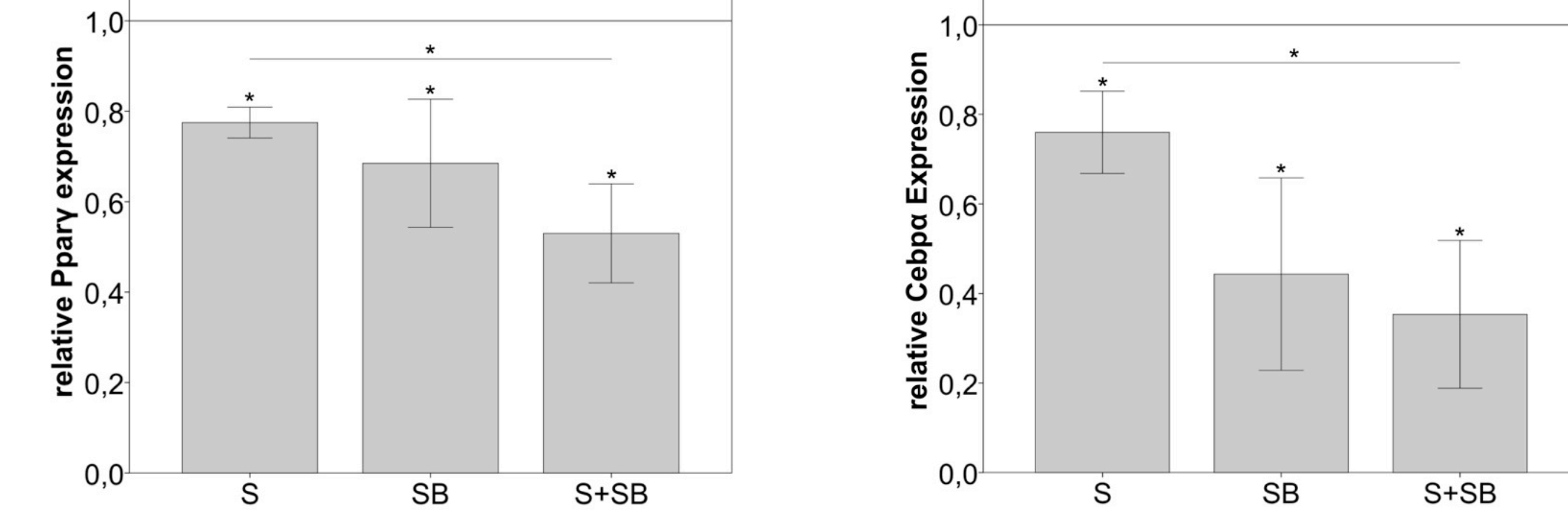

Mechanical loading and SB415286 treatment increased the gene expression of Wnt10b, which promotes osteoblastogenesis. SB415286 treatment in addition to mechanical loading enhanced the mechanically induced expression of Wnt10b.
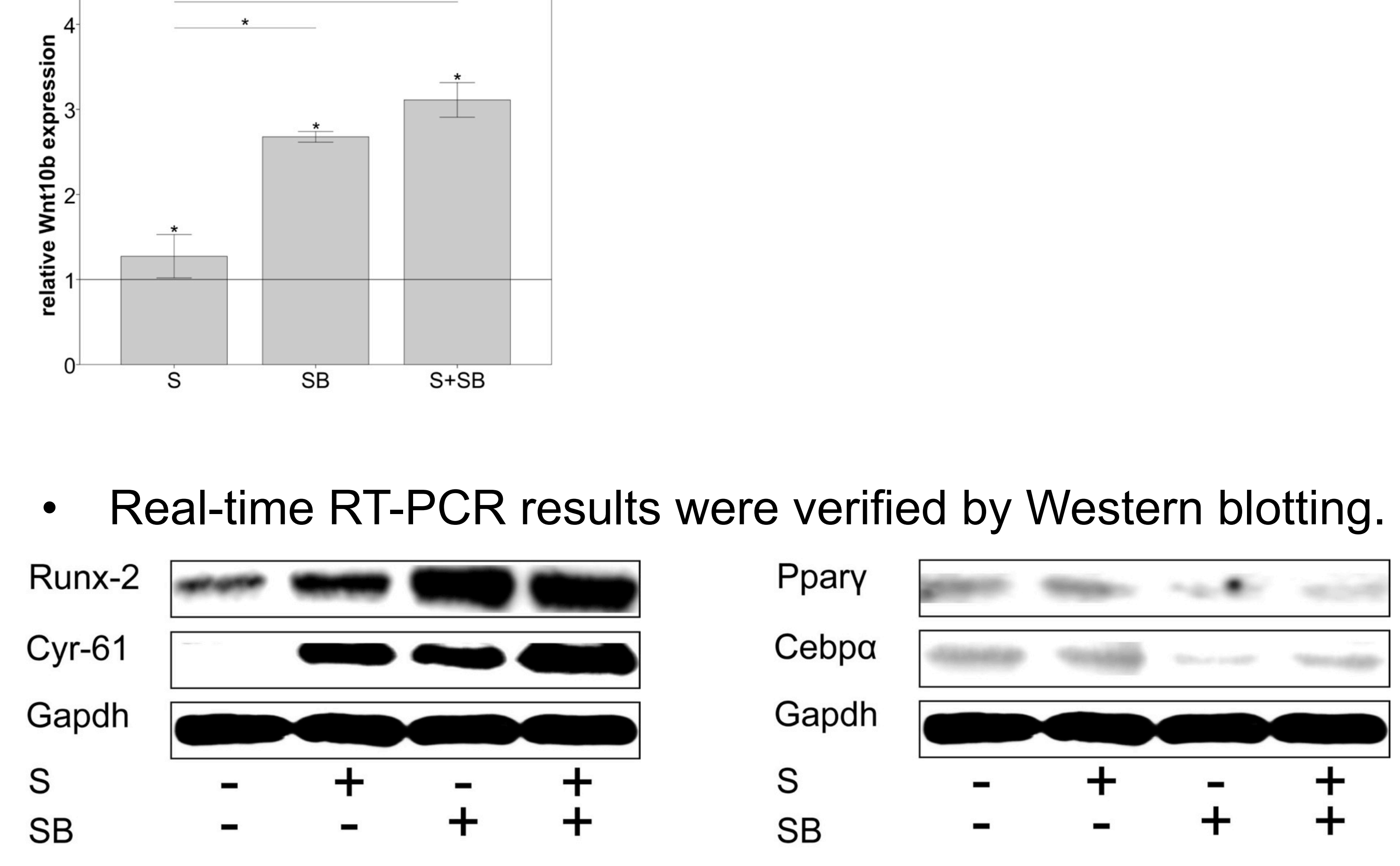

\section{Conclusions}

Activation of $\beta$-catenin signalling and mechanical loading led to a reduction of lipid vacuoles in $\mathrm{C} 3 \mathrm{H} 10 \mathrm{~T} 1 / 2$ cells cultivated under adipogenic conditions. Activation of $\beta$ catenin signalling in addition to mechanical loading completely abolished lipid vacuole formation.

Activation of $\beta$-catenin signalling and mechanical loading led to an increase of osteogenic marker expression and to a reduction of adipogenic marker expression in C3H10T1/2 cells. Activation of $\beta$-catenin signalling seemed to have a sensitizing effect on the mechanically induced osteogenic gene expression as well as on the mechanically reduced adipogenic gene expression.

Interestingly, the expression of Wnt10b, which is known as an inhibitor of adipogenesis and a stimulator of osteoblastogenesis [2], was upregulated by $\beta$ catenin signalling activation and mechanical loading.

Sensitizing mechanosensitive pathways, which contribute to the enhancement of osteogenesis and simultaneous impairment of adipogenesis, might represent a therapeutic option for osteoanabolic therapy in patients with osteoporosis.

\section{Acknowledgements}

This study was kindly supported by the German Research Foundation (DFG, IG 18/5-2). None of the authors has any conflicts of interest.

\section{References}

11] Justesen et al, Biogerontology 2 (2001) 165-171

2] Christodoulides et al, Trends Endocrinol Metab 20 (2009) 16-24

[3] Case and Rubin, J Cell Biochem 110 (2010) 545-553

[4] Robinson et al, J Biol Chem 281 (2006) 31720-31728 